\title{
Potential involvement of Drosophila flightless-1 in carbohydrate metabolism
}

\author{
Jung-Eun Park ${ }^{1, \#}$, Jinho Jang ${ }^{2, \#}$, Eun Ji Lee ${ }^{1}$, Su Jung Kim ${ }^{3}$, Hyun Ju Yoo ${ }^{3}$, Semin Lee, ${ }^{2, *} \mathcal{E}$ Min-Ji Kang, ${ }^{1, *}$ \\ ${ }^{1}$ Department of Biomedical Sciences, University of Ulsan College of Medicine, Asan Medical Center, Seoul 05505, ${ }^{2}$ Department of \\ Biological Engineering, School of Life Sciences, Ulsan National Institute of Science and Technology, Ulsan 44919, ${ }^{3}$ Department of \\ Convergence Medicine, Asan Institute for Life Sciences, Asan Medical Center, University of Ulsan College of Medicine, Seoul 05505, Korea
}

\begin{abstract}
A previous study of ours indicated that Drosophila flightless-1 controls lipid metabolism, and that there is an accumulation of triglycerides in flightless-1 (fli)-mutant flies, where this mutation triggers metabolic stress and an obesity phenotype. Here, with the aim of characterizing the function of Flil in metabolism, we analyzed the levels of gene expression and metabolites in flil-mutant flies. The levels of enzymes related to glycolysis, lipogenesis, and the pentose phosphate pathway increased in flil mutants; this result is consistent with the levels of metabolites corresponding to a metabolic pathway. Moreover, high-throughput RNA sequencing revealed that Drosophila Flil regulates the expression of genes related to biological processes such as chromosome organization, carbohydrate metabolism, and immune reactions. These results showed that Drosophila Flil regulates the expression of metabolic genes, and that dysregulation of the transcription controlled by Flil gives rise to metabolic stress and problems in the development and physiology of Drosophila. [BMB Reports 2018; 51(9): 462-467]
\end{abstract}

\section{INTRODUCTION}

Flightless-1 was originally identified in Drosophila (1) and has been described as an actin-remodeling protein that belongs to the gelsolin protein superfamily (2-4). Flightless- 1 contains a gelsolin-like actin-binding domain at the $C$ terminus whereas a leucine-rich repeat (LRR) domain is located at the $\mathrm{N}$ terminus (1). The LRR domain is involved in both intramolecular

*Corresponding authors. Semin Lee, Tel: +82-52-217-2663; Fax: +82-52-217-2299; E-mail: seminlee@unist.ac.kr; Min-Ji Kang, Tel: +82-2-3010-2797; Fax: +82-2-3010-5307; E-mail: mjkang@amc. seoul.kr

${ }^{\text {"}}$ These authors contributed equally to this work.

https://doi.org/10.5483/BMBRep.2018.51.9.153

Received 10 July 2018, Revised 24 July 2018, Accepted 25 July 2018

Keywords: Flightless-1, Gene expression, Metabolism, Metabolomics, RNA sequencing recognition and structural organization. The gelsolin like domain mediates actin-binding and protein-protein interactions. The Flightless protein has been implicated in actin filament organization during cell migration and tissue repair (5). Beyond cytoskeletal function, flightless- 1 has been shown to act as a transcriptional coregulator that can either positively or negatively affect the activity of transcription factors (6-9). Flightless-1 interacts with nuclear receptor (NR), transcription coactivators, and the SWI/SNF chromatin-remodeling complex. Flightless-1 binds to BAF53, a component of $\mathrm{SWI} / \mathrm{SNF}$ complexes, as well as to estrogen receptor $\alpha(E R \alpha)$, thus contributing to the recruitment of SWI/SNF complexes to the promoter of ER $\alpha$ targets (10). In addition, flightless-1 forms a complex with NR coactivators-glucocorticoid receptorinteracting protein 1 (GRIP1) and coactivator-associated arginine methyltransferase 1 (CARM1) - which leads to the enhancement of NR function (6). In contrast, flightless-1 inhibits $\beta$-catenin-mediated transcription through interfering with the binding of FLII leucine-rich repeat-associated protein 1 (FLAP1) to p300 and $\beta$-catenin (8).

A recent study of ours has shown that Drosophila flightless-1 (Flil) plays a role in lipid metabolism (11). Drosophila flil mutants show increased levels of triglycerides and are resistant to starvation. In that study, the upregulation of Flil suppressed the mRNA expression of Desaturase-1, and conversely, the mRNA expression of Desaturase-1 increased in flil-mutant flies, suggesting that Drosophila Flil downregulates Desaturase-1 at the transcriptional level, thereby contributing to the obese phenotype of flil mutants. Based on these results, our purpose here was to investigate the genes whose expression levels are regulated by flightless-1 in Drosophila, thereby contributing to the above phenotype. Specifically, we examined the expression levels of genes related to energy metabolism, lipogenesis, and lipolysis; these genes may contribute to the obese phenotype, specifically, the increased levels of triglycerides and insulin resistance, in flil-mutant flies. The mRNA expression of most glycolytic-enzyme genes and of some lipogenic enzymes was found to be specifically higher in flil-mutant flies. Accordingly, we found that the levels of stearoyl-coenzyme A $(\mathrm{COA})$ and palmitoyl-CoA specifically increased in flil mutants according to metabolomic analysis. In addition, high-throughput RNA

ISSN: 1976-670X (electronic edition)

Copyright (C) 2018 by the The Korean Society for Biochemistry and Molecular Biology

(c) This is an open-access article distributed under the terms of the Creative Commons Attribution Non-Commercial License (http://creativecommons.org/licenses/by-nc/4.0) which permits unrestricted non-commercial use, distribution, and reproduction in any medium, provided the original work is properly cited. 
sequencing (RNAseq) analysis of flil-mutant flies revealed that flightless-1 regulates the expression of genes related to chromatin organization, carbohydrate metabolic process, and proteolysis. Taken together, these results support the transcriptional role of flightless- 1 in the expression of genes associated with metabolism.

\section{RESULTS}

\section{Metabolic reprogramming in flil mutants}

Our recent study indicates that Drosophila flightless-1 mutant flies contain large amounts of triglycerides, which contribute to starvation resistance (11). In general, fat accumulation has been primarily attributed to food intake and energy expenditure $(12,13)$. Based on the fact that our previous results revealed that flil mutant flies do not consume more food (11), we tested whether or not Flil is required for the gene expression related to energy metabolism. To that end, we compared the transcript levels of glycolytic genes between seven-day-old controls and flii ${ }^{3 / 14}$ mutants. These flil mutant alleles, $f|i|^{3}$ and $f l i l^{14}$, have been characterized in previous studies $(11,14,15)$. The $\mathrm{flil}^{3}$ mutant allele has a single-base substitution of Gly to Ser at amino acid position 602 , which is homozygotically viable. In contrast, the $\mathrm{flil}^{14}$ allele is lethal during the larval and pupal stages. The mRNA expression of most glycolytic-enzyme genes, including HexA, Pgi, Gapdh, $P g k$, and Eno, specifically increased in flil $^{3 / 14}$ mutants. Among them, the $A / d$ transcript was upregulated more than sixfold in flil $^{3 / 14}$ mutant flies, while the mRNA expression of $L d h$ significantly decreased (Fig. 1A). In contrast, the mRNA expression of tricarboxylic acid (TCA) cycle genes, CG7430 and Scsa, did not significantly increase (Fig. 1B). These results showed that the mutation of flil in Drosophila accelerated glucose metabolism throughout the body. 6-Phosphogluconate dehydrogenase (Pgd) in the pentose phosphate pathway, which is essential for the supply of NADPH (16), was also

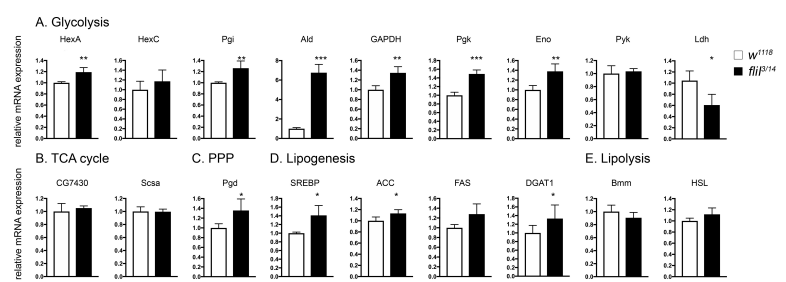

Fig. 1. The changes in expression of metabolic genes in flil mutants. Quantitative RT-PCR analysis of each gene in seven-day-old $w^{1118}$ flies (controls) and flil $^{3 / 14}$ mutants. The values were normalized to Rp49 data. (A) Relative mRNA abundance of glycolytic-enzyme genes. (B) Relative mRNA expression of TCA cycle genes. (C) Relative expression of Pgd mRNA. (D) Relative expression levels of lipogenic genes. (E) Relative mRNA expression of lipolytic genes. Data are presented as mean $\pm \mathrm{SE}$ from at least five independent experiments; ${ }^{* P}<0.05, * * P<$ 0.01 , and $* * * P<0.001$ upregulated in $\mathrm{flil}^{3 / 14}$ mutant flies (Fig. 1C). The byproduct of NADPH produced by 6-Phosphogluconate dehydrogenase activity may be utilized for lipid synthesis. In addition, we found that the mRNA expression of lipogenic genes, such as sterol-regulatory element-binding protein (SREBP) and its downstream target genes, fatty acid synthase (FAS) and acetyl-CoA carboxylase (ACC), was slightly higher in $\mathrm{flil}^{3 / 14}$ mutants (Fig. 1D). In line with these data, we found that the mRNA expression of DGAT1, which catalyzes the conversion of diacylglycerol and fatty acyl CoA to triglycerides, was significantly increased (Fig. 1D). In contrast, the mRNA expression levels of lipolytic genes, brummer $(B m m)$ and hormone-sensitive lipase (HSL), did not significantly change (Fig. 1E). Altogether, these results suggest that Flil is necessary for the regulation of glucose metabolism and the transcription of lipogenic genes.

\section{Metabolomic analysis of flil mutants reveals a change in precursors of long-chain fatty acids}

In order to understand how changes in gene expression

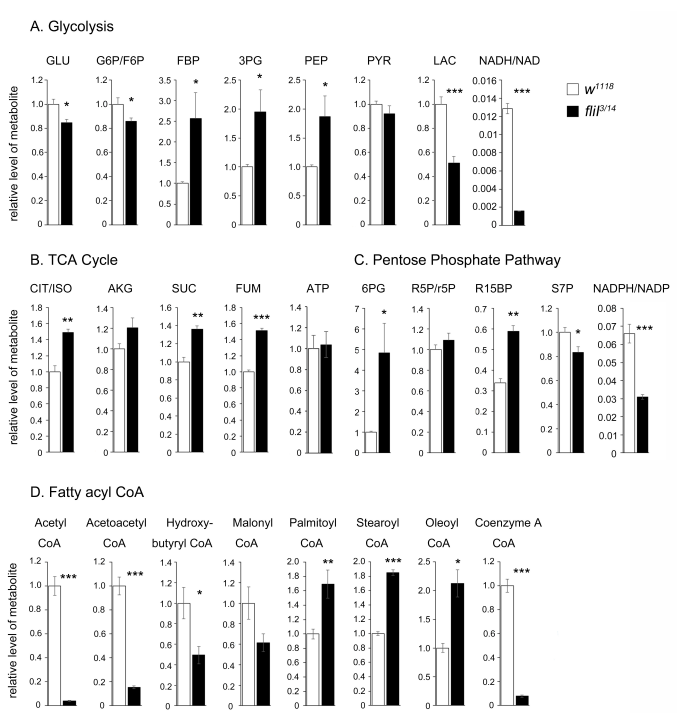

Fig. 2. Flil mutants show increased levels of precursors of long-chain fatty acids. The metabolites from seven-day-old $w^{1118}$ flies (controls) and $\mathrm{flil}^{3 / 14}$ mutants were monitored by LC-MS/MS. (A) The amounts of intermediates of glycolysis. The values of each metabolite amount were normalized to the total protein level. (B) The relative levels of intermediates of the TCA cycle. These amounts significantly increased in $\mathrm{fli}^{3 / 14}$ mutants compared to $\mathrm{w}^{1118}$ flies, the controls. (C) The levels of intermediates of the pentose phosphate pathway (PPP). The ratio of NADPH/NADP involving a byproduct of the PPP, which was strongly diminished in $\mathrm{flil}^{3 / 14}$ mutant flies. The relative levels of fatty acyl CoAs are illustrated in (D). The amounts of precursors of long-chain fatty acyl-CoAs, such as palmitoyl-CoA, stearoyl-CoA, and oleoyl-CoA, were considerably larger in $\mathrm{flil}^{3 / 14}$ mutants relative to $w^{1118}$ flies (controls). Data are presented as mean $\pm \mathrm{SE}$ from at least three independent experiments; $* \mathrm{P}<0.05, * * \mathrm{P}<0.01$, and $* * * \mathrm{P}<0.001$. 
influence fat metabolism in flil mutants, we compared the metabolic profiles of control and flil $^{3 / 14}$ flies through mass spectrometry.

Specifically, we examined the intermediates of glycolysis, of the TCA cycle, of the pentose phosphate pathway, and the coenzymes involved in the metabolism of fatty acids. In agreement with the results of mRNA expression analysis by quantitative PCR, two important metabolic pathways, glycolysis and the TCA cycle, differed between $w^{1118}$ flies (controls) and $\mathrm{flil}^{3 / 14}$ mutants (Fig. 2A, B). Although lactate was shown to be downregulated in $\mathrm{flil}^{3 / 14}$ mutants, the amounts of glycolysis intermediates, such as fructose-1,6-bisphosphate, 3-phosphoglycerate, and phosphoenolpyruvate, increased relative to controls. Flil ${ }^{3 / 14}$ mutants also manifested slight but significant increases in the amounts of TCA cycle intermediates, namely citrate/isocitrate, succinate, and fumarate. Nevertheless, the total ATP level did not differ between the two groups. In addition, we found that a pentose phosphate pathway intermediate, 6-phosphogluconate, was significantly upregulated in $\mathrm{flil}^{3 / 14}$ mutant flies. The amount of ribulose-1,5-bisphosphate, which is formed from ribulose 5-phosphate in the cooperative pentose phosphate pathway, significantly increased in $\mathrm{fli}^{3 / 14}$ mutant flies. A previous study suggests that ribulose-1,5bisphosphate stimulates phosphofructokinase- 1 and inhibits its opposing enzyme, fructose 1,6-bisphosphatase (17); this mechanism may contribute to glycolysis and the pentose phosphate pathway. NADPH is generated by the pentose phosphate pathway and is utilized for fatty acid synthesis. $\mathrm{Flil}^{3 / 14}$ mutants also manifested a significant reduction in the ratio of NADPH/NADP (Fig. 2C).

The accumulation of long-chain acyl CoAs is frequently observed in obesity or type 2 diabetes $(18,19)$. Given that $\mathrm{flil}^{3 / 14}$ mutants had an obesity-like phenotype, we tested whether this phenotype in $\mathrm{flil}^{3 / 14}$ mutants directly affects the amounts of long-chain acyl CoAs. Although seven-day-old flii $^{3 / 14}$ mutants showed decreased levels of coenzyme A and short-chain acyl CoA compared to the wild type, long-chain acyl CoAs, such as palmitoyl-CoA, oleoyl-CoA, and stearoyl-CoA, were found to be upregulated in $\mathrm{fli}^{3 / 14}$ mutant flies (Fig. 2D).

Differences in the expression levels of metabolic genes and relevant metabolites between $w^{1118}$ flies (control) and flil $^{3 / 14}$ mutants, as well as the changes in flit $^{3 / 14}$ mutants are summarized in Fig. 3. In particular, increased levels of mRNA or a metabolite in $\mathrm{flil}^{3 / 14}$ mutants are labeled in red, downregulation is indicated with blue, and unchanged ones are shown in black.

Given that Drosophila flightless-1 represses the expression of Desaturase-1 (11), whose preferred substrates are palmitoyl$\mathrm{CoA}$ and stearoyl-CoA, we believe that the elevated amounts of long-chain acyl CoAs contributed to the obesity-like phenotype of $\mathrm{flil}^{3 / 14}$ mutant flies.

\section{Drosophila flightless-1 regulates the expression of genes related to chromatin remodeling}

In order to gain a genome-wide view of the changes in gene expression specifically induced by flightless-1, we performed a differentially expressed gene (DEG) analysis of RNA-seq data from seven-day-old $w^{118}$ control and fliil/14 adult flies. Only

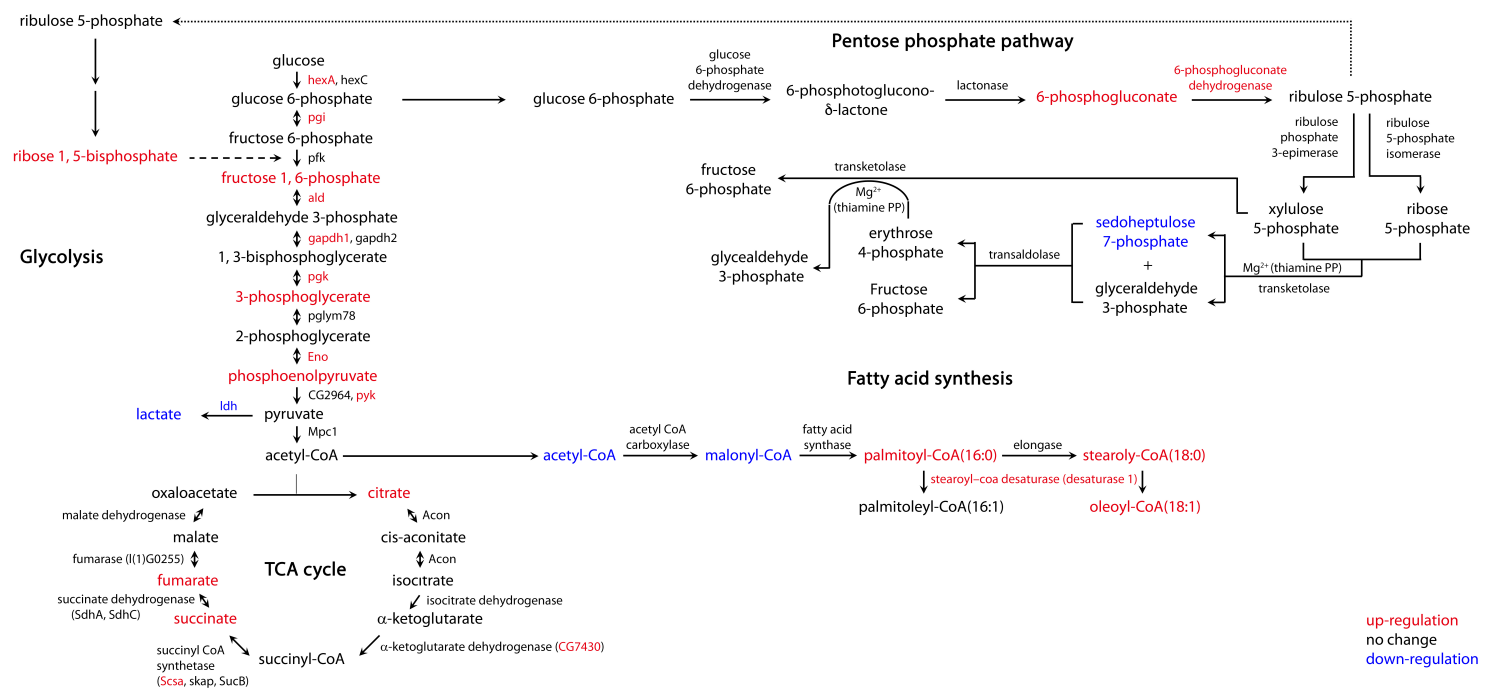

Fig. 3. A schematic diagram of glucose metabolism and lipid metabolism according to the data from Figs. 1 and 2 . The red color indicates that mRNA or metabolite levels were significantly upregulated in terms of statistics in flil ${ }^{3 / 14}$ mutants, the blue color denotes downregulation in flil $^{3 / 14}$ mutants, and black indicates no change. 
Table 1. GO terms of genes regulated in flightless-1 mutant flies

\begin{tabular}{|c|c|c|c|}
\hline GO term & Pvalue & Benjamini & Genes \\
\hline \multicolumn{4}{|l|}{ Downregulated } \\
\hline $\begin{array}{l}\text { GO:0051276 - chromosome } \\
\text { organization }\end{array}$ & $3.98 \times 10^{-7}$ & $7.44 \times 10^{-5}$ & $\begin{array}{l}\text { CG33822, CG33810, CG33825, RHI, CG33816, CG33828, } \\
\text { CG33861, CG33831, CG33858 }\end{array}$ \\
\hline $\begin{array}{l}\text { GO:0031057 - negative regulation of } \\
\text { histone modification }\end{array}$ & $8.97 \times 10^{-7}$ & $8.39 \times 10^{-5}$ & CG33822, CG33810, CG33825, CG33816, CG33828, CG33831 \\
\hline GO:0006334 - nucleosome assembly & $1.58 \times 10^{-6}$ & $9.87 \times 10^{-5}$ & $\begin{array}{l}\text { CG33822, CG33810, CG33850, CG33825, CG33816, } \\
\text { CG33828, CG33861, CG33844, CG33831, CG33858, } \\
\text { CG33847 }\end{array}$ \\
\hline $\begin{array}{l}\text { GO:0005975 - carbohydrate metabolic } \\
\text { process }\end{array}$ & $2.70 \times 10^{-6}$ & $1.26 \times 10^{-4}$ & $\begin{array}{l}\text { MAL-A7, MAL-A6, TOBI, MAL-A8, AMY-P, MAL-A1, AMY-D, } \\
\text { AMYREL, CHT8, MAL-B1 }\end{array}$ \\
\hline GO:0006342 - chromatin silencing & $1.39 \times 10^{-4}$ & $5.19 \times 10^{-3}$ & $\begin{array}{l}\text { CG33822, CG33810, CG33850, CG33825, CG33816, } \\
\text { CG33828, CG33844, CG33831, CG33847 }\end{array}$ \\
\hline \multicolumn{4}{|l|}{ Upregulated } \\
\hline GO:0006508 - proteolysis & $3.74 \times 10^{-9}$ & $7.29 \times 10^{-7}$ & $\begin{array}{l}\text { CG9897, CG32523, ANCE-4, SPH93, CG42694, CG8539, } \\
\text { CG1304, CG17239, SER6, CG15254, CG11842, GAMMATRY, } \\
\text { CG9676, CG31267, CG7829, CG31681, CG3088, CG14529, } \\
\text { ZETATRY, CG17475, CG8329, CG11911 }\end{array}$ \\
\hline $\begin{array}{l}\text { GO:0050830 - defense response to } \\
\text { Gram-positive bacterium }\end{array}$ & $1.53 \times 10^{-5}$ & $1.49 \times 10^{-3}$ & LYSP, LYSE, LYSD, SPH93, LYSB, GNBP1, IM23 \\
\hline GO:0019835 - cytolysis & $9.95 \times 10^{-5}$ & $6.45 \times 10^{-3}$ & LYSP, LYSE, LYSD, LYSB \\
\hline $\begin{array}{l}\text { GO:0016998 - cell wall macromolecule } \\
\text { catabolic process }\end{array}$ & $2.32 \times 10^{-4}$ & $1.13 \times 10^{-2}$ & LYSP, LYSE, LYSD, LYSB \\
\hline
\end{tabular}

the transcripts with adjusted $\mathrm{P}$ values (Benjamini-Hochberg correction) $<0.05$ and a fold change $>2$ in gene expression between the two groups were selected for subsequent Gene Ontology (GO) enrichment analysis. According to this criterion, 181 genes were downregulated in flil mutants, while 160 genes were upregulated (see Table S1). The Gene Ontology (GO) terms with adjusted $\mathrm{P}<0.05$ are listed in Table 1. Specifically, GO terms enriched in the downregulated genes included those involved in chromosome organization (GO:0051276, adjusted $P$ value of $7.44 \times 10^{-5}$ ), the negative regulation of histone modification (GO:0031057, adjusted $\mathrm{P}$ value of $8.39 \times 10^{-5}$ ), nucleosome assembly (GO:0006334, adjusted $\mathrm{P}$ value of $9.87 \times 10^{-5}$ ), and a carbohydrate metabolic process (GO:0005975, adjusted $P$ value of $1.26 \times$ $10^{-4}$ ). This indicates that Drosophila Flil not only regulates specific gene expression related to glucose metabolism, but also seems to regulate the gene expression in general under certain conditions by reorganizing chromatin structure. Prominent among the genes whose expression increased significantly were those encoding proteolytic enzymes (GO:0006508, adjusted $\mathrm{P}$ value of $7.29 \times 10^{-7}$ ), proteins related to a defense response to a gram-positive bacterium (GO:0050830, adjusted $\mathrm{P}$ value of $1.49 \times 10^{-3}$ ), and proteins associated with cytolysis (GO:0019835, adjusted $P$ value of $6.45 \times 10^{-3}$ ). The activators of Imd signaling (PGRP-LC) and lysozymes (LysB, -D, and -E) were highly expressed in flil mutant flies (Table S1). An abnormally active immune response is associated with the upregulation of stress response factors, all of which have been previously linked to immune function, including the translational repressor 4E-BP/Thor (20). These data are in agreement with those of our recent study suggesting that the mRNA level of 4E-BP is increased in $\mathrm{flil}^{3 / 14}$ mutants (11). The RNA-seq. results were further validated through quantitative real-time PCR (Supplemental Fig. 2). These observations indicate that the transcriptional activity of flightless-1 controls the expression of genes related to chromatin remodeling, metabolism, and immune responses.

\section{DISCUSSION}

Here, we reveal the transcriptional influence of Drosophila Flil on the expression of genes including those participating in chromatin remodeling, carbohydrate metabolic processes, and immune responses. Unexpectedly, our RNAseq analysis suggests that Drosophila flightless-1 regulates the expression of histones, which are basic nuclear proteins that are responsible for the nucleosome structure of the chromosomal fiber (21, 22). Histones are required for the condensation of nucleosome chains into higher-order structures. They also function as regulators of individual gene transcription through chromatin remodeling. In addition, the genes whose expression decreased in flightless-1 mutants, rhino and CREG, are known to control gene transcription through producing Piwi-interacting RNA (23) or inhibiting transcription factor binding (24). Thus, although Drosophila flightless-1, as a transcription factor, may regulate the expression of genes related to specific pathways 
including glucose metabolism, immune responses, or proteolysis, it seems to regulate gene expression by reorganizing the chromatin structure under certain conditions. These phenomena need to be validated through further studies.

Given that $\mathrm{flil}^{3 / 14}$ mutants show features of a metabolic disorder (11), we analyzed the changes in metabolites in the metabolic pathways including glycolysis, the TCA cycle, pentose phosphate pathway, and fatty acid synthesis (Fig. 2). Through this assay, we found that the NADPH/NADP ratio was clearly lower in the mutants. NADPH is an essential cofactor in de novo lipogenesis, which requires NADPH as a reducing agent for the conversion of acetyl-CoA into fatty acids (25-27). The amounts of NADPH in flil ${ }^{3 / 14}$ mutants were relatively low as compared to those in $w^{1118}$ flies. We can explain this result as a form of supply and demand: NADPH is mainly generated by the pentose phosphate pathway. Our results indicate that the level of ribulose 1,5-phosphate, which is a product of the pentose phosphate pathway, significantly increased in $\mathrm{flil}^{3 / 14}$ mutant flies (Fig. 2). In addition, mRNA expression of the gene encoding one of the NADPH-producing enzymes, phosphogluconate dehydrogenase (Pgd), increased slightly but significantly in flil mutants (Fig. 1C). Therefore, we can theorize that in $\mathrm{flil}^{3 / 14}$ mutants, NADPH and acetyl-CoA were rapidly consumed for the elongation of long-chain fatty acids, driving the increase in fat contents throughout the body. Among the intermediates of glycolysis, the amount of lactate significantly decreased in $\mathrm{flil}^{3 / 14}$ mutant flies; this is consistent with the observed reduction in Ldh mRNA expression. As shown in Fig. 3, the levels of expression of glycolytic genes and glycolysis intermediates were significantly increased in $\mathrm{flil}^{3 / 14}$ mutant flies compared to in control flies. However, the mRNA expression levels of tricarboxylic acid (TCA) cycle genes and the levels of TCA cycle intermediates were only slightly changed. Thus, we believe that the increased amounts of glycolytic intermediates were not consumed for lactate production but were instead mostly converted to acetyl-CoA for the elongation of long-chain fatty acids.

Given that $\mathrm{flil}^{3 / 14}$ mutant flies showed increased levels of triglycerides, we expected that the amounts of lipogenic enzymes, such as SREBP, FAS, and ACC, would be greater in $\mathrm{flil}^{3 / 14}$ mutant flies, but there were slight and significant differences in the expression of these proteins between $w^{1118}$ and $f\left(i l^{3 / 14}\right.$ flies. Emerging evidence indicates that the activity of a metabolic enzyme can be regulated post-translationally under certain conditions without changing the expression level (28-30). We assumed that an enzyme's activity can somewhat contradict its expression level because enzymes may be regulated by post-translational modifications in the mutant. Therefore, in future studies, it is necessary to test the activities of the enzymes that we examined in this study.

In summary, we analyzed the expression levels of metabolic genes and the levels of metabolites in flil-mutant flies. These results support previous findings showing that Drosophila Flil serves as a key regulator of lipid metabolism. In addition,
RNAseq analysis revealed the transcriptional targets of Flil in Drosophila, which include the genes related to chromosome organization, carbohydrate metabolism, proteolysis, and immune responses.

\section{MATERIALS AND METHODS}

\section{Plasmids and fly strains}

All Drosophila stocks were raised at $25^{\circ} \mathrm{C}$ on a standard cornmeal medium containing $4.94 \%$ molasses, $3.8 \%$ cornmeal, $1.6 \%$ yeast, and $1.2 \%$ agar. Genes were expressed in Drosophila through the standard Gal4/UAS system. Fly strains $w^{1118}$ (stock number 5905), flil ${ }^{3}$ (stock number 4730), and fli $^{14} /$ FM6 (stock number 7481) were obtained from the Bloomington Stock Center.

\section{Quantitative RT-PCR}

Total RNA was isolated from five female flies using the TRIzol Reagent (Invitrogen, USA), and 200 ng of RNA was transcribed using the ReverTra Ace qPCR RT Kit (Toyobo Co., Japan). Quantitative PCR amplification was run for 40 cycles by means of the TOPreal ${ }^{T M}$ qPCR $2 X$ PreMIX (SYBR Green with high ROX) and a LightCycler ${ }^{\mathbb{R}} 480$ Real-Time PCR System. Rp49 served as a reference for normalization. Relative quantification of mRNA was performed through the comparative $\mathrm{C}_{\mathrm{T}}$ method (31). The primers are listed in Supplemental Table 2. Detailed information is included in the Supplemental Material.

\section{ACKNOWLEDGEMENTS}

This work was supported by the National Research Foundation (2015R1C1A2A01051560 to M.J.K.) and the Asan Institute for Life Sciences (2018-577 to M.J.K.).

\section{CONFLICTS OF INTEREST}

The authors have no conflicting interests.

\section{REFERENCES}

1. Campbell HD, Schimansky T, Claudianos C et al (1993) The Drosophila melanogaster flightless-I gene involved in gastrulation and muscle degeneration encodes gelsolin-like and leucine-rich repeat domains and is conserved in Caenorhabditis elegans and humans. Proc Natl Acad Sci U S A 90, 11386-11390

2. Archer SK, Behm CA, Claudianos C and Campbell HD (2004) The flightless I protein and the gelsolin family in nuclear hormone receptor-mediated signalling. Biochem Soc Trans 32, 940-942

3. Ghoshdastider U, Popp D, Burtnick LD and Robinson RC (2013) The expanding superfamily of gelsolin homology domain proteins. Cytoskeleton (Hoboken) 70, 775-795

4. Nag S, Larsson M, Robinson RC and Burtnick LD (2013) Gelsolin: the tail of a molecular gymnast. Cytoskeleton 
(Hoboken) 70, 360-384

5. Cowin AJ, Adams DH, Strudwick XL et al (2007) Flightless I deficiency enhances wound repair by increasing cell migration and proliferation. J Pathol 211, 572-581

6. Lee YH, Campbell HD and Stallcup MR (2004) Developmentally essential protein flightless I is a nuclear receptor coactivator with actin binding activity. Mol Cell Biol 24, 2103-2117

7. Wu L, Chen H, Zhu Y et al (2013) Flightless I homolog negatively regulates ChREBP activity in cancer cells. Int J Biochem Cell Biol 45, 2688-2697

8. Lee YH and Stallcup MR (2006) Interplay of Fli-I and FLAP1 for regulation of beta-catenin dependent transcription. Nucleic Acids Res 34, 5052-5059

9. Choi JS, Choi SS, Kim ES et al (2015) Flightless-1, a novel transcriptional modulator of PPARgamma through competing with RXRalpha. Cell Signal 27, 614-620

10. Jeong KW, Lee YH and Stallcup MR (2009) Recruitment of the SWI/SNF chromatin remodeling complex to steroid hormone-regulated promoters by nuclear receptor coactivator flightless-I. J Biol Chem 284, 29298-29309

11. Park JE, Lee EJ, Kim JK, Song Y, Choi JH and Kang MJ (2018) Flightless-I Controls Fat Storage in Drosophila. Mol Cells 41, 603-611

12. Church C, Moir L, McMurray F et al (2010) Overexpression of Fto leads to increased food intake and results in obesity. Nat Genet 42, 1086-1092

13. Bray GA and Popkin BM (1998) Dietary fat intake does affect obesity! Am J Clin Nutr 68, 1157-1173

14. de Couet HG, Fong KS, Weeds AG, McLaughlin PJ and Miklos GL (1995) Molecular and mutational analysis of a gelsolin-family member encoded by the flightless I gene of Drosophila melanogaster. Genetics 141, 1049-1059

15. Perrimon N, Smouse D and Miklos GL (1989) Developmental genetics of loci at the base of the $X$ chromosome of Drosophila melanogaster. Genetics 121, 313-331

16. Frederiks WM, Bosch KS, De Jong JS and Van Noorden CJ (2003) Post-translational regulation of glucose-6-phosphate dehydrogenase activity in (pre)neoplastic lesions in rat liver. J Histochem Cytochem 51, 105-112

17. Ishikawa E, Ogushi S, Ishikawa T and Uyeda K (1990) Activation of mammalian phosphofructokinases by ribose 1,5-bisphosphate. J Biol Chem 265, 18875-18878

18. Zlobine I, Gopal K and Ussher JR (2016) Lipotoxicity in obesity and diabetes-related cardiac dysfunction. Biochim Biophys Acta 1860, 1555-1568

19. Bandyopadhyay GK, Yu JG, Ofrecio J and Olefsky JM (2006) Increased malonyl-CoA levels in muscle from obese and type 2 diabetic subjects lead to decreased fatty acid oxidation and increased lipogenesis; thiazolidi- nedione treatment reverses these defects. Diabetes 55, 2277-2285

20. Bernal A and Kimbrell DA (2000) Drosophila Thor participates in host immune defense and connects a translational regulator with innate immunity. Proc Natl Acad Sci U S A 97, 6019-6024

21. Andersen JS, Lam YW, Leung AK et al (2005) Nucleolar proteome dynamics. Nature 433, 77-83

22. Clapier CR, Iwasa J, Cairns BR and Peterson CL (2017) Mechanisms of action and regulation of ATP-dependent chromatin-remodelling complexes. Nat Rev Mol Cell Biol $18,407-422$

23. Zhang Z, Wang J, Schultz N et al (2014) The HP1 homolog rhino anchors a nuclear complex that suppresses piRNA precursor splicing. Cell 157, 1353-1363

24. Veal E, Eisenstein M, Tseng ZH and Gill G (1998) A cellular repressor of $\mathrm{E} 1 \mathrm{~A}$-stimulated genes that inhibits activation by E2F. Mol Cell Biol 18, 5032-5041

25. Lin R, Elf S, Shan C et al (2015) 6-Phosphogluconate dehydrogenase links oxidative PPP, lipogenesis and tumour growth by inhibiting LKB1-AMPK signalling. Nat Cell Biol 17, 1484-1496

26. Zimmermann R, Haemmerle G, Wagner EM, Strauss JG, Kratky D and Zechner R (2003) Decreased fatty acid esterification compensates for the reduced lipolytic activity in hormone-sensitive lipase-deficient white adipose tissue. J Lipid Res 44, 2089-2099

27. Al-Dwairi A, Pabona JM, Simmen RC and Simmen FA (2012) Cytosolic malic enzyme 1 (ME1) mediates high fat diet-induced adiposity, endocrine profile, and gastrointestinal tract proliferation-associated biomarkers in male mice. PLoS One 7, e46716

28. Petridou A, Chatzinikolaou A, Avloniti A et al (2017) Increased Triacylglycerol Lipase Activity in Adipose Tissue of Lean and Obese Men During Endurance Exercise. J Clin Endocrinol Metab 102, 3945-3952

29. Stofkova A, Krskova K, Vaculin S and Jurcovicova J (2016) Enhanced activity of hormone sensitive lipase (HSL) in mesenteric but not epididymal fat correlates with higher production of epinephrine in mesenteric adipocytes in rat model of cachectic rheumatoid arthritis. Autoimmunity 49, 268-276

30. Kim SJ, Tang T, Abbott M, Viscarra JA, Wang $Y$ and Sul HS (2016) AMPK Phosphorylates Desnutrin/ATGL and Hormone-Sensitive Lipase To Regulate Lipolysis and Fatty Acid Oxidation within Adipose Tissue. Mol Cell Biol 36, 1961-1976

31. Choi SW, Lee KS, Lee JH et al (2016) Suppression of Akt-HIF-1 $\alpha$ signaling axis by diacetyl atractylodiol inhibits hypoxia-induced angiogenesis. BMB Rep 49, 508-513 